\title{
From the Editor: A New Home for Technostyle
}

As some of our CATTW members know, a year ago Technostyle began its search for a new home. This was a timely decision, but those of us who have worked on the journal at Douglas College will miss the Technostyle experience.

Editing Technostyle has been a most rewarding professional experience for me, and I have deeply appreciated the opportunity that the CATTW Executive and membership have afforded me in carrying out the responsibilities of this position. I have enjoyed a productive association with all those who have collaborated with us over the last seven years: they have all taught me a great deal. This includes not only our contributors, but also a very able and flexible cadre of reviewers (too numerous to name here), our translators (Jacqueline Bossé-Andrieu, Marie-Claire Chinniah, Céline Beaudet, and Zélie Guével), a very resourceful and competent sequence of Print Futures Professional Writing students at Douglas College, and members of the Editorial Board (especially Janet Giltrow and Lilita Rodman who have provided invaluable consultation when called upon for their expert advice).

So, while I will miss my involvement with Technostyle, I believe it is time for others to reap the benefits from the same opportunity that I have had. It is with great pleasure and faith that we pass responsibility for the journal on to very able faculty at the University College of Fraser Valley (UCFV) - Faye Hyndman, Gloria Borrows, and Nadeane Trowse. We have also made happy arrangements to continue to provide the editorial team with Douglas College professional writing students who will complete their practicums as managing editors.

This issue of Technostyle is thus the last to be produced at Douglas College. Somehow it seems fitting that at this juncture of change two of the founding members of CATTW have articles in this issue. Jacqueline Bossé-Andrieu documents grammatical differences between French and English that translators need to take into account when writing in both languages, and Michael Jordan examines theories of relevance to establish five major elements of relevance - textual and communicative - that offer professional communicators (specifically technical writers), teachers, and theorists a "useful concept" for practice. We are also happy to welcome a new voice to the journal and association, Shannon Kelly, whose critical discourse analysis marshalls Fairclough's triadic model of discourse functions to examine how government documents on university funding utilize "the language of control" to ensure its own agenda prevails. 
Each of these articles makes an important contribution to scholarship of interest and use to CATTW members. Our readers should also find the four book reviews in this issue of interest - they address social and ethical issues in technical writing (Dombrowski); managing technical writing classroom projects (Hackos and Lanyi); lay and expert attitudes toward linguistic usage (Cameron); and the rhetoric of everyday texts (Stillar). Technostyle's review editor, Janet Giltrow, and assistant, Shurli Makmillen, who ensure these reviews are ready for each issue, will thankfully be part of the new team at UCFV.

I wish to extend special appreciation to our current managing editors, Sharon Russell and Eden Evans, two professional writing students who took on their duties with a zeal and conscientiousness that made my job a breeze. They have not only managed and produced this current issue, but have also produced the impressive collection that Randy Harris edited in the previous issue. I would like to commend them as exemplary professionals, and to congratulate them on their graduation from the Print Futures Professional Writing program

We hope you enjoy this issue, and we wish our UCFV colleagues a happy and productive sojourn with Technostyle.

Diana Wegner, Ph.D

English \& Communications

Douglas College

P.O. Box 2503

New Westminster, BC V2L 5B2 


\section{Message de la rédactrice en chef : Technostyle se relocalise}

Comme certains membres de l'ACPRTS le savent déjà, depuis un an, nous étions à la recherche d'un nouveau lieu d'accueil pour Technostyle. À titre de rédactrice en chef de la revue, j'ai bénéficié d'une expérience professionnelle très enrichissante et je suis très redevable au conseil d'administration et aux membres de l'ACPRTS qui m’ont donné cette chance. Pendant sept ans, j'ai apprécié l'efficace contribution de tous les collaborateurs de notre publication. Grâce à eux, j'ai beaucoup appris. Parmi ces personnes, je compte non seulement les auteurs, mais aussi les réviseurs, très compétents et très ouverts (trop nombreux hélas pour que je puisse les énumérer tous ici), nos traducteurs (Jacqueline Bossé-Andrieu, Marie-Claire Chinniah et Céline Beaudet), les cohortes successives d'étudiants en rédaction professionnelle du programme Print Futures de Douglas College, - toujours compétents et pleins de ressources - , ainsi que les membres du comité de rédaction (en particulier Janet Giltrow et Lilita Rodman, qui m'ont toujours donné des conseils précieux lorsque j'ai fait appel à leur expertise).

Je quitte la direction de la revue non sans regret, mais je crois que le moment est venu de permettre à d'autres de bénéficier à leur tour des avantages associés à cette fonction. C'est donc avec grand plaisir et beaucoup de confiance que je cède la responsabilité de Technostyle à la très compétente équipe de la University College of Fraser Valley (UCFV) : Faye Hyndman, Gloria Borrows et Nadeane Trowse. Grâce à l'heureuse entente que nous avons conclue ensemble, les étudiants en rédaction professionnelle de Douglas College continueront à participer à la rédaction de la revue dans le cadre de stages.

Le présent numéro est donc le dernier à être produit à Douglas College. En cette période de transition, il sied bien que deux des membres fondateurs de l'ACPRTS, Jacqueline Bossé-Andrieu et Michael Jordan, y publient des articles. La première traite des différences grammaticales entre le français et l'anglais dont les traducteurs doivent tenir compte lorsqu'ils rédigent dans l'une ou l'autre langue. Le second examine les théories de la pertinence en vue de déterminer cinq caractères pertinents fondamentaux, du point de vue du texte et de la communication, fournissant ainsi aux communicateurs professionnels (en particulier les rédacteurs techniques), aux formateurs et aux chercheurs un outil conceptuel utile pour la pratique. Nous sommes

également heureux d'accueillir un nouvel auteur et un nouveau membre de l'ACPRTS en la personne de Shannon Kelly, qui nous présente une analyse de discours critique 
en canalisant les trois fonctions du discours du modèle de Fairclough : l'article montre comment une administration publique documente les subventions universitaires en utilisant un langage de contrôle qui lui permet de mettre de l'avant son propre programme.

Chacun de ces articles constitue une importante contribution à la recherche; les membres de l'ACPRTS devraient à la fois y trouver intérêt et utilité. La présente livraison contient aussi quatre intéressantes recensions de livres portant sur les thèmes suivants : questions éthiques et sociales en rédaction technique (Dombrowski); gestion de projets de rédaction technique effectués en classe (Hackos and Lanyi); attitudes des experts et des profanes à l'égard de l'usage linguistique (Cameron); et rhétorique des textes de tous les jours (Stillar). La responsable de la section des comptes rendus, Janet Giltrow, et son assistante Shurli Makmillen, qui voient à ce que les recensions soient prêtes à temps pour chaque numéro, continueront à faire ce travail pour la nouvelle équipe de la UCFV, ce dont on ne peut que se réjouir.

Je tiens à exprimer tous mes remerciements à nos deux rédacteurs actuels, Sharon Russell and Eden Evans, deux étudiants en rédaction professionnelle qui ont pris leurs fonctions très à cœur et avec beaucoup de dynamisme, facilitant ainsi grandement mon travail. Outre la rédaction et la production de ce numéro, ils ont participé à l'impressionnant collectif réuni par Randy Harris, qui a constitué le numéro antérieur. Je tiens à souligner leurs compétences exemplaires et à les féliciter pour l'obtention de leur diplôme dans le programme Print Futures en rédaction professionnelle.

Nous nous souhaitons une bonne lecture. Quant à nos collègues de la UCFV, qui prennent la relève dans la production de Technostyle, nos meilleurs vœux de succès et de plaisir dans l'entreprise les accompagnent !

Diana Wegner, Ph.D

English \& Communications

Douglas College

P.O. Box 2503

New Westminster, BC V2L 5B2 